\section{Clinical decision making by dentists working in the NHS General Dental Services since April 2006}

\author{
B. J. B. Davies ${ }^{1}$ and F. Macfarlane ${ }^{2}$
}

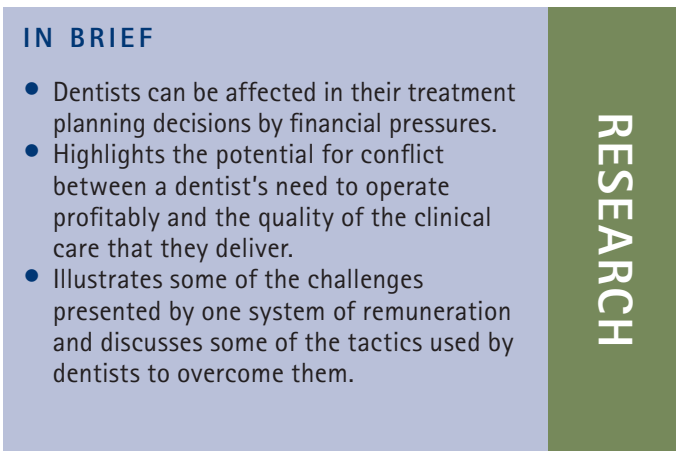

\begin{abstract}
IIn April 2006 a new contract was introduced that governed how NHS General Dental Practitioners would be funded for the services they provide. This study looks at the impact that the contract has had in the three years since its introduction, evaluating its influence on the clinical care that patients receive and the clinical decisions that dentists are making. This qualitative service evaluation involved interviewing 12 dentists representative of a range of NHS dentists involved with the new NHS dental contract using a semi-structured approach. We found evidence that the new contract has led to dentists making different decisions in their daily practice and sometimes altering their treatment plans and referral patterns to ensure that their business is not disadvantaged. Access to care for some patients without a regular dentist can be compromised by the new contract as it can be financially challenging for a dentist to accept to care for a new patient who has an unknown and potentially large need for treatment. Cherry-picking of potentially more profitable patients may be common. The incentive is to watch borderline problems rather than to treat if a treatment band threshold has already been crossed and treatment may be delayed until a later course of treatment for the same reason. Dentists often feel that complex treatments (for example, endodontic treatments) are financially unviable. Some dentists are referring difficult cases that might previously have been treated 'in house', such as extractions, to another provider, as this enables offloading of costs while potentially retaining full fees. Younger and less experienced dentists may be further pressured.
\end{abstract}

\section{INTRODUCTION}

The introduction in April 2006 of a new NHS General Dental Services (nGDS) contract has been widely commented upon, most recently in a wide-ranging review of NHS dentistry commissioned by the United Kingdom Department of Health. ${ }^{1}$ The aim of this research was to explore whether this new contract influenced clinical decisions made by dentists.

The nGDS contract was implemented as outlined in Options for change. ${ }^{2}$ It was described as the greatest reform of state funded dental care since the inception of the NHS, introduced the Unit of Dental Activity (UDA) and promised a

Specialist in Oral Surgery, Defence Dental Services, Dental Centre, Scotton Road, Catterick Garrison, North Yorkshire, DL9 3LD; ${ }^{2}$ Senior Lecturer in Health Care Management, School of Management, University of Surrey, Guildford, Surrey, GU2 7XH

*Correspondence to: Mr Benedict Davies Email: bjbdavies@googlemail.com

Online article number E17

Refereed Paper - accepted 8 July 2010

DOI: 10.1038/sj.bdj.2010.1080

${ }^{\circ}$ British Dental Journal 2010; 209: E17 shift towards preventive based dental care. Dentists, as independent contractors to the NHS, have a need to operate at a profit and it has been suggested ${ }^{3}$ that the wide range of treatments that fall into each charge band place financial and ethical pressure on the dentist.

The House of Commons Health Committee Report of $2008^{4}$ offered extensive evidence that complex treatments are becoming less common in general practice under the nGDS contract. The Department of Health argues in its evidence to the Report that this is because appropriate treatment is being provided now that the financial incentives to provide complex treatments available under the previous item-of-service type of contract have been removed. The Report observes however that there is no evidence to support this claim and offers that the reverse situation may be equally true with complex treatment not being offered when it is most appropriate due to inadequate remuneration.

The literature is lacking in quantitative and qualitative research regarding the effects of the new contract on clinical care, perhaps due to the short timeframe since inception of the contract. The stated intention of Options for change $e^{2}$ was that the new GDS contract would encourage a high quality of dentistry and increase the provision of preventive care and advice.

The evidence accumulated by the Health Committee Enquiry ${ }^{4}$ strongly suggests that the contract is failing in this. It is suggested that dentists are failing to give preventive advice, the number of complex treatments such as crowns and root canal treatments has fallen (by 45\% since 2004, according to evidence presented to the Health Committee Enquiry) and that the number of extractions has increased. The same Enquiry was concerned by an apparent increase in referrals of patients requiring complex treatment to hospitals and PCT salaried dental services.

The aim of the Department of Health's NHS dentistry: delivering change ${ }^{5}$ review was 'to build an NHS dental service that offers access to high quality treatment for patients when they need to see a dentist, 
focuses on preventing disease, ...gives a fair deal to dentists and their teams and improves their working lives'. The dental profession was however sceptical about these aims ${ }^{6}$ with a widespread belief that changes were politically motivated and a majority feeling that neither working lives nor patient care would be improved. ${ }^{7}$

The 2007 BDA NHS new contract survey ${ }^{8}$ of dentists showed that 93\% of respondents thought that the new system had not encouraged a more preventive approach to care. The BDA's research also showed that dentists' confidence in the future of NHS dentistry had fallen dramatically. 95\% of respondents said that they were less confident about the future of NHS dentistry than was the case two years ago.

When an nGDS dentist refers the care of a patient to a secondary provider, the care is provided at no cost to the referring dentist. National NHS payment by results tariffs for referral-based treatment provided in a secondary care setting is typically higher by a factor of many multiples than the UDA-based fee that an nGDS dentist would receive for providing the same care. Typically, dentists meet the majority of their patients' treatment needs 'in house', referring only patients with more complex dental problems. There is however likely to be some variance in referral patterns with some dentists referring disproportionately more or less. The causes of these variations do not seem to be explained in the literature although may be suggested to include not just ability and competence but also the financial (dis)incentives for providing the treatment implicit in the method of remuneration.

There is no set standard for what dentists should refer and what treatments they are expected to personally perform. Being prescriptive with referral guidelines may risk pushing clinicians into treatment that they do not feel competent to perform. It is however difficult to be certain or dogmatic about the motivation to refer patients with currently only anecdote suggesting that referral decisions are not always based solely on a dentist's 'knowledge, professional competence and physical abilities, ...when it is necessary, or if the patient asks.'

\section{METHODS}

The study was an exploratory study employing qualitative techniques to establish the views of dentists involved in the nGDS contract. The interviews were open-ended and semi-structured, lasting 30 to 60 minutes. Each interviewee was asked to comment openly on the nGDS contract and how it exerts influence on patient care. Participants were encouraged to focus on clinical decisions taken in general practice. Data were thematically analysed using 'The Framework Approach'10 allowing themes to inductively emerge from the data. ${ }^{11}$ Each transcript was summarised into thematic areas before being cross-tabulated against other interviews to facilitate comparative analysis. A qualitative interview-based approach was employed as 'interviews can offer unique insight into people's' personal perspectives, providing a more comprehensive understanding of their beliefs, knowledge and attitudes as well as offering greater depth and methodological flexibility than quantitative research methods such as structured questionnaires.' ${ }^{12}$

This study fulfilled the criteria to be considered a service evaluation for the purposes of ethical approval and does not therefore need to be subject to NHS ethics review. ${ }^{13}$ Written consent was sought from all research participants including permission to record and transcribe interviews respecting participants' rights to anonymity where requested and/or where their identity is immaterial to the data.

The sample was a non-probability heterogeneous purposive group of 12 interviewees chosen from registered UK dental practitioners involved in NHS dental services with experience from 3 to 40 years. This included dentists from different sectors of the profession including those working as principals and associates in the NHS GDS, dentists working in representative and regulatory positions at both a local and a national level, dentists working in salaried positions, teaching positions, specialist dentists and dentists working in the hospital sector. Several of the interviewees were able to represent multiple viewpoints due to their roles in different capacities across the profession. Examples include interviewees who combine responsibilities such as teaching, salaried or hospital roles with NHS GDS and / or representative / regulatory commitments. Interviews took place between August and December 2008.

\section{Findings}

Overall, the interviews highlighted several major themes in referral patterns and decision-making. These included low incentives for preventive work; game-playing to maximise utility from the new contract; changes in treatment profiles offered; a move towards shorttermism in care planning; reduction in the threshold of referral to secondary care; and a trend towards 'cream-skimming' of patients. Interviewees agreed that none of this was ideal for individual patients or the population as a whole. They felt that the new contract had introduced a real tension between what was needed for financial viability of practices and what would benefit the patient.

Each emerging theme will be explored in detail.

\section{Impact on preventive care}

There was agreement among the respondents that the new contract offered little incentive for preventive interventions such as health education and periodontal treatments. The contract was felt to 'offer no incentive to do preventive work at all, because it is not accounted for in the UDA system. Many interviewees wanted to do more prevention and several interviewees admitted to encouraging patients to see a dental hygienist on a privately funded basis.

\section{Game-playing}

It was felt to be 'inevitable that the drive in the new contract is towards doing the least you can get away with in the category band: One interviewee stated that while over-prescription represented a small minority of dentists working in the old system, 'perverse incentives in the new contract will encourage a far greater cohort to under-prescribe' as dentists sought to do the minimum amount that they could to cross a UDA treatment band threshold. Game-playing and interpreting rules literally was felt to be inevitable when dentists are running businesses and are going to see a substantial loss by providing some treatment over others.

\section{Altered treatment profiles}

Most interviewees agreed that the push was definitely more towards more simple treatment plans and that the new contract 
had certainly stopped the 'drill and fill' of old. Whilst there was agreement that this had potential to be a positive shift with the new contract dissuading unnecessary treatment, it was felt that the balance between over- and under-treatment has swung too far towards under-treatment. Several interviewees felt that in borderline cases they were more likely to suggest an extraction where previously endodontic treatment may have been offered. Another interviewee felt that he was 'just being more robust about which teeth had a reasonable restorative prognosis. Most of the interviewees were inclined to acknowledge that while they were aware of such issues, they believed that their personal practice was relatively unchanged. Other interviewees said that they might, for example, try to encourage a private endodontic referral.

All interviewees acknowledged that sometimes the most appropriate treatment (such as extensive prosthetic work or endodontic treatment) is 'totally financially unjustifiable' so less appropriate treatment may be encouraged and it was agreed that both statistically and anecdotally there had been a proportionally 'vast increase in simple treatments' and a comparable decrease in complex treatment. Treatments that had been routine under the old contract such as bitewing radiographs to screen for caries were said to be less likely because 'in the old system you were getting a fiver and now you get nothing so why spend the extra time?' and any disease found on them would be a financial liability requiring treatment. Another interviewee added that there was a 'subconscious incentive to leave borderline stuff or address more major stuff first' and an inclination to 'monitor more and be less invasive'.

\section{Short-termism}

There was a recurring theme among many of the interviewees that the approach that a dentist takes to their treatment planning is associated with their long-term intention to stay at the same workplace. A dentist planning on staying in a practice in the long term may be prepared to take a shortterm loss to develop a 'good' long-term patient who requires only simple maintenance. In such dentists there is more of an incentive to employ a more preventive approach so that future disease is easier to manage. For a dentist with more short- term intentions, the inclination may be to do the minimum possible to get over the UDA treatment band threshold.

\section{Negative impact on referral patterns}

There was almost universal agreement that there are incentives to refer treatment under the nGDS. Several explanations were offered for what was described by one interviewee as a 'vast increase in referrals':

- Cost-shifting - dentists are at risk of financial loss if they complete some courses of treatment for 3 UDAs so will look to 'offload' the patient or aspects of their care to reduce the liability of care that they must provide while retaining the full UDA value. An interviewee cited examples of dentists who are 'suddenly unable to treat patients that they have been successfully treating for years because of spurious medical problems or phobias.' One interviewee explained that 'if you have a large treatment plan and you can refer on aspects of that treatment plan while still being paid the same fee then you are much more likely to refer' while another explained that 'extractions are not practice builders and if you don't get paid for it then why would you do it?'

- Training standards and clinician competence - a widely expressed thought was that the increase in referrals was not simply about the new contract but also a large influence was a gradual change in the ability of dentists to provide what was once seen as routine care. It was generally agreed that dentists trained in recent years had less competence in areas such as basic oral surgery than the dentists of previous decades. Several interviewees recognized this, feeling that it is difficult and potentially dangerous for patients if referrals for basic treatment from dentists who may not have the ability to provide the care are refused. It was agreed that 'it is wrong to force dentists to practice beyond their competency'; however, it was suggested by one interviewee that there was a need for a defined basic skill standard that NHS dentists must reach.

- Changing demographic of GDS practitioners - a change in the demographic of NHS practice was also felt to contribute to increased referrals with significant numbers of experienced dentists moving away from the NHS and being replaced by younger and/or non-UK graduates who were felt to be potentially less experienced both clinically and of NHS practice.

\section{Reduced incentives to provide band three treatments}

There was extensive comment that treatments falling into UDA treatment band 3 were largely unviable despite attracting 12 UDAs. The magnitude of the patient's element of the fee of $£ 198$ was considered to put patients off if it was for only one or two teeth whereas dentists were less likely to be prepared to treat more than 1 or 2 units for 12 UDAs. The expense of laboratory bills was felt to negate a lot of the potential revenue in 12 UDAs. There was said to be 'substantial danger of making a loss in providing band 3 treatments', especially 'if they do not go right first time', with one interviewee calling it 'financial suicide to do lab work!'.

\section{Barriers to registering new patients}

New patients were described as getting a 'particularly poor deal'. The nGDS contract was felt to be 'grossly unfair' on dentists treating high numbers of new patients and it was agreed that starting a new list was difficult, especially in areas where disease levels and patients failing to attend appointments may be higher.

Many practices wanted to avoid taking new patients as they represent an unknown liability that may saddle the business with a substantial loss once the responsibility to treat the patient has been accepted. Several interviewees expressed regret at this, feeling that they have 'to be ruthless for the sake of their business but feel sorry for the patients' who were unable to access care.

\section{Splitting treatment plans and cost implications}

There was evidence that dentists were splitting treatment plans and this was considered to be inevitable. This was described as doing just enough to get into the UDA band and then 'watching' other diseased but asymptomatic teeth 'for a few months when it's a new course of treatment' and 
claiming a further 3 UDAs. Such teeth may have been treated immediately under an 'item-of-service' system of funding where the drive may be to treat earlier.

\section{'Cherry-picking' patients}

There was anecdotal evidence of dentists 'cherry-picking' the patients that they can treat financially viably on the NHS. It was suggested to occur by several means:

- By doing a private check up and then offering to treat patients under GDS terms.

- Offering to see family members of existing 'good' patients.

- Seeing a patient for urgent treatment but refusing them continuing care once aware that they are likely to be a financial liability.

\section{Impact on quality}

There was widespread feeling that the nGDS contract has not improved the quality of patient care. Whilst it was accepted that there is currently 'no good evidence to support this', the 'driver was generally towards sub-optimal treatment'. This was acknowledged to be hard to prove, although examples were offered where dentists delay treatment until disease is at a more advanced stage with the resulting treatment being ultimately more destructive to the tooth.

\section{DISCUSSION}

With advances in dental care, the distinction between treatment that is necessary to secure oral health and what might be considered as perhaps cosmetic or ideal becomes ever more challenging. This demands realism and clarity of what services must be offered under NHS terms. Dentists currently risk making substantial losses by providing one treatment option over another and so, consciously or subconsciously, may advocate for example, extracting a tooth that might otherwise be retained with a root canal filling and crown if the financial implication of doing so were not so damaging.
The new contract appears to have failed to deliver improvements in the delivery of preventive care as dentists do not receive financial recognition of their time investment. Some dentists may see it as in their long term interest to minimise the disease liability that they will be expected to treat on their regular patients; however, the increasing itinerancy of both the dental workforce and dental patients detracts from this long term perspective. This may be compounded by the loss of patient registration which means that dentists may not have the assurance that a short-term investment in preventive measures will be returned by longer-term patient loyalty. There was a widespread desire among dentists to operate preventively and the potential incorporation into the contract of additional payments to recognize preventive practice was felt to be a welcome incentive.

The nGDS contract allows a dentist to accept a patient for treatment and refer on elements of the treatment that they feel unable to provide. A treatment plan that offers a dentist 3 UDAs such as one involving multiple fillings and extractions will still generate 3 UDAs for the dentist if elements (such as the extractions) of that treatment plan are passed on to another facility. Not only does referral incur no cost to the referring dentist but it allows the dentist to offload a financial liability that they would otherwise face.

Whilst PCTs do specify the core services that dentists must provide under the new contract, in the majority of cases, there are no robust standards of what it is acceptable to refer. Responsibility in many areas for refusing referrals rests with the secondary provider who accepts the referrals and who could have a financial incentive to accept referrals for basic care that could be provided by the primary care practitioner. It is however also difficult for a secondary provider to refuse a referral without robust guidelines as the assumption that a referrer may be offloading costs could be misplaced. They may be genuinely unable to provide that care by virtue of training or equipment and refusing the referral could push a dentist into performing inappropriate or inadequate treatment, thus compromising patient safety.

Whilst there appears to be universal agreement that the old contract was unsatisfactory for a multitude of reasons and desperately in need of reform, the general sentiment from the dental profession seems to be that its replacement has presented some new and different issues that appear to be impacting on patient care.

Overall, the majority of interviewees were happy to cite anecdotal examples of the new contract creating questionable practices. However, they were generally keen to reinforce that in their personal practice their work is within the terms of the contract. This is laudable but arguably unsurprising, as an admission to the contrary would leave the dentist exposed to the risk of official censure. With such a reluctance for interviewees to admit to first hand experiences of their decisions being influenced, it was therefore necessary to draw on the anecdotal examples as probably representative if they were recounted by multiple sources.

1. Department of Health. NHS dental services in England: an independent review by Professor Jimmy Steele. London: Department of Health, 2009.

2. Department of Health. NHS dentistry: options for change. London: Department of Health, 2002.

3. Milsom K M, Threlfall A, Pine K, Tickle M, Blinkhorn A S, Kearney-Mitchell P. The introduction of the new dental contract in England - a baseline qualitative assessment. Br Dent J 2008; 204: 59-62.

4. House of Commons Health Committee. Dental services. London: House of Commons Health Committee, 2008.

5. Chief Dental Officer. NHS dentistry: delivering change. London: Department of Health, 2004.

6. Hudson S. A fistful of UDAs. Br Dent J 2007; 203: 71-73.

7. Baird W O Jackson $\mathrm{R} J$, Worthington LS, Robinson $P$ G. Perspectives of dentists in primary care ahead of the 'new ways of working'. Br Dent J 2007; 202: 614-615.

8. British Dental Association. New NHS contract survey. London: British Dental Association, 2007.

9. Standards for dental professionals. London: General Dental Council, 2005.

10. Ritchie J, Spencer L. Qualitative data analysis for applied policy research. In Bryman A, Burgess R (eds) Analysing qualitative data. London: Routledge, 1994

11. Dawson C. A practical guide to research methods. 3rd ed. Oxford: How To Books, 2007.

12. Stewart $K$, Gill $P$, Chadwick $B$, Treasure E. Qualitative research in dentistry. Br Dent J 2008; 204: 235-239.

13.National Research Ethics Service. Differentiating audit, service evaluation and research. London: NRES Ethics Consultation e-group; 2007. 